\section{New way of seeing}

\author{
Brandy Schillace
}

The cover of our September issue features a detail from Jacques Fabien Gautier d'Agoty's Mylologie complette. I have had the good fortune to see many of Gautier's works at the Dittrick Museum of Medical History, and the even better luck of teaching courses through their use. The students might read Frankenstein (a work preparing to celebrate its 200th birthday), and they might see anatomies of the body online, or listen to me speak at length about the value of early anatomists to the medical future. But it wasn't until our hands-on class, where white-gloved students could see the highly coloured Gautier in person, that the message became clear. One of the anatomies displays the body in full, high colour, still deeply penetrating-and seven feet in length. The hushed whisper around the room: it's a person, isn't it? The body, only yet in two-dimensions, became real.

This is a colourful example of something we, from both sides of medical humanities, tend to take for granted. How do we see the body? A simple question on the surface but endlessly complex. What do we mean by the body? Whose body is it? Or to turn it around-who gets to see it? And do we trust their vision? In "Deal with It. Name It':the diagnostic moment in film', Thierry Jutel and Annemarie Jutel discuss the 'crisis' of diagnosis using film, a literal and figurative lens. What we call something makes it real to us. On the reverse, though related side, 'The Ghost of Pandemics Past: Revisiting Two Centuries of Influenza in Sweden' by Martin Holmberg (and open access in this issue) describes the way people connect with the concept of 'pandemic' by either contrasting it to the 'novelty of the coming plague' or recast it as fear of a 'ghost-like repetition of the past'. Being unable to name and understand what a pandemic constitutes results in ahistorical accounts and reduces communities' ability to prepare and resist. Fear may be a motivator, but dread of the ambiguous or unknown is a stalemate. How we see bodies of evidence, how we see human bodies and how we envision ourselves, our future and our ability to shape it hinges on widening the

Correspondence to Dr Brandy Schillace, Dittrick Medical History Center and Museum, Case Western Reserve University, Allen Memorial Medical Library, 3rd Floor, 11000 Euclid Ave, Cleveland, Ohio 44106-1714, USA; bls10@case.edu lens but without losing precious focus. And this, at the beginning of my tenure as Medical Humanities new editor in chief, is my mission. Many changes will be forthcoming in the journal, but always with the aim of seeing more and with greater clarity.

Our upcoming December issue on Shame, Stigma and Medicine, will be the last issue under our previous submission protocol and article types. In 2018, we will be making a number of relevant and timely changes, including a revision of our mission statement-which will appear on a soon-to-be updated and restyled journal home page. To wit:

Medical Humanities is a leading international journal that offers a platform not only for presenting, but also for shaping the conversation around medicine as practice and philosophy as it engages with humanities and arts, social sciences, health policy, medical education, patient experience and the public at large. We seek scholarly and critical submissions on subjects of interest, including: epidemics and disease, history of medicine, cultures of medicine, disability studies, gender and the body, communities in crisis, bioethics, and public health as they inform and are informed by the humanities.

BMJ's Medical Humanities has always endeavoured to reflect the 'whole field' of medical humanities. Building on this and the wise stewardship of our previous editors and editorial board, we now seek to shape that conversation as well. Breadth has been a challenge as well as a boon to our field, with some defining medical humanities as principally for practical use in medical education and by clinicians. Others have considered medical humanities as primarily cultural representations of health in a wide variety of literatures. Meanwhile, emphasis in the USA has shifted increasingly towards bioethics, health and society. The value of our broad lens is that we welcome all of these perspectives, seeing in them the whole of medical humanities, a field that at its core considers the human story behind and within medicine, its history, its cultural valence and its influence on practice.

At the same time, however, 'medical' as a moniker has its own complexities. For many, 'medicine' may mean the institutionalised profession of medicine in the USA and the UK. Medical Humanities will, in its remit, take a different perspective. Western biomedicine is not the oldest nor even the largest means of health treatment. From Cambodia, India and China to vast stretches of Africa and South America, from indigenous tribes to alternative practices within cities, the promotion of health, from our perspective, constitutes 'medicine'. Similarly, my work with medical museums in the USA, UK and Europe has taught me that the history of medicine is, in reality, the story of all our long struggle for human health. Just as a museum-goer to the Dittrick will encounter nursing, midwifery, public health and physical therapy, as well as the history of access to care, a reader of Medical Humanities should expect to find diverse and disparate means of health practice. We welcome works that use the methods of the humanities and social sciences to critically engage with this expanded view of medicine. Likewise, we welcome the view from medical practitioners as they thoughtfully engage with the humanities as a means of inquiry into their own practice, pedagogy and medicine in general. The relevant question is not merely what we practice, but how, for whom and why.

Importantly, medical humanities is a means of reflection on and examination of biomedicine in context-and a recognition that context is politicised, culturally complex and frequently ambiguous. Seeing so broad a vista requires an interdisciplinary approach. To aid in shaping what has become a robust and diverse field requires rigorous, critical and engaged research. We know that you, our readers, our authors, are the backbone of such an endeavour. We know, too, that medical humanities work has been ongoing in other disciplines, sometimes without calling itself as such-including work in the life sciences and public health, history of medicine and health geography. We welcome you to join us and to make Medical Humanities a sounding voice in a changing field. Most importantly, we want to offer points of connection between and among scholars, and to facilitate that dialogue, we offer new categories for submission to both the journal and the Medical Humanities blog.

Medical Humanities will publish only peer-reviewed content, through a doubleblind submission, in four categories: Original Research (increased from 5000 to 9000 words), Current Controversies (shorter articles that deal with a specific divisive topic), Commentaries and Review Essays. For those who are familiar with the journal, there are a few conspicuous absences. The journal will not be 
publishing poetry as a stand-alone item in either the print or online version of the journal after December. We will continue to welcome research articles that discuss and describe poetry (and art and film) in the context of medical humanities, and seek to expand scholarly engagement on the subject. Another change involves the removal of the student essay as a category, but not because we want to discourage student submission-far from it. Instead, we hope the contraction of our submission categories will help focus the journal's scope. Academically rigorous, robust, cutting edge (and even edgy) works will always be welcome here, and we look forward to reading them and to sharing them. The Medical Humanities blog will also be offering new and revised categories for submission, including interviews, brief reports (on current issues) and 'provocations', or short posts on points of contention and discussion in the field presently. The blog will continue to solicit and provide book reviews and also to feature guest posts on thematic issues generally including global health and film and media studies. Look for new guidelines on the website for each article type.

Medical Humanities offers a platform for true interdisciplinarity-for those working at the intersections of fields for connection rather than mere multiplication of foci. The future of our field lies in dialogue, a place to share ideas and to move them, to shake and shape them and finally to bring them forward to a wider public. We seek disciplinary rigour-and open minds. We seek to engage, not merely to tell. We want the works that fight fires, and those that start them, works unafraid to engage with controversy and unapologetic in the support of bioethics. And we welcome those from beyond the UK and USA to help us expand the vision. Medical Humanities is, as it has always been, reflecting the 'whole field' but with renewed emphasis on critical, academic and rigorous scholarship. Together with an engaged readership, we seek to bridge the distance that has sometimes appeared between the history of medicine, science, technology and healthcare and the medical humanities and to provide a platform for dialogue-our body of work, seen with new eyes.

In the rest of this September issue, we feature works on bioethics and imagination; about arts-based training and empathy, chronic pain, face transplant and social justice; and about the ways we position ourselves through literature and narrative vis-à-vis artists like van Gogh or memoirs by Arthur Frank and Kathlyn Conway. I invite you also to investigate the literature review on narrative medicine and compassionate care, a letter about labels and self-injury and the poems in print and online that fill out this, Medical Humanities 43rd issue-and my first. In each reading, I hope you will find a new perspective, knowing that though we are leaving many of these categories behind, each of them has provided light for us to see by, an illumination for which I am eternally grateful. To Deborah, the outgoing editor in chief, my endless thanks. To you, the reader, my thanks and a standing invitation.

\section{Competing interests None declared.}

Provenance and peer review Commissioned; internally peer reviewed.

(C) Article author(s) (or their employer(s) unless otherwise stated in the text of the article) 2017. All rights reserved. No commercial use is permitted unless otherwise expressly granted.

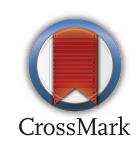

To cite Schillace B. Med Humanit 2017;43:139-140. Accepted 2 August 2017

Med Humanit 2017;43:139-140. doi:10.1136/medhum-2017-011341 